\title{
Effects of gender and aging in patients who undergo coronary artery bypass grafting: From the FU-Registry
}

\author{
Tomohiko Shimizu ${ }^{1}$, Shin-ichiro Miura ${ }^{1}$, Kazuma Takeuchi², \\ Tadashi Tashiro ${ }^{2}$, Keijiro Saku ${ }^{1}$ \\ ${ }^{1}$ Department of Cardiology, Fukuoka University School of Medicine, Fukuoka, Japan \\ ${ }^{2}$ Department of Cardiovascular Surgery, Fukuoka University School of Medicine, Fukuoka, Japan
}

\begin{abstract}
Background: It is unclear whether gender and aging influence the characteristics of patients who undergo coronary artery bypass grafting (CABG).

Methods: We retrospectively reviewed a clinical database of 1,498 patients (male/female = $=1133 / 365$, age $67 \pm 9$ years) who underwent CABG at Fukuoka University Hospital from 1994 to 2010.
\end{abstract}

Results: Male showed significantly younger, higher percentages (\%) of smoking and hyperuricemia (HU), higher levels of serum creatinine, and lower \% hypertension (HT) and diabetes mellitus (DM), and lower levels of left ventricular ejection fraction than female. In multivariate analysis, all parameters identified independent variables associated with the gender difference. Next, we divided the patients into 5 groups according to age, and each group was then separated by gender. The \% of males significantly decreased with aging, whereas $\%$ female significantly increased. Although \% smoking and estimated glomerular filtration rate (eGFR), and body mass index (BMI) in all patients, males and females significantly decreased with aging, $H U$, left ventricular end diastolic pressure and the number of significantly stenosed coronary vessels were not associated with gender or aging. Interestingly, \% HT in all patients and males significantly increased with aging, whereas that in females was not associated with aging. Serum low-density lipoprotein cholesterol levels in males significantly decreased with aging, while those in all patients and females were not associated with aging. In this contemporary data set, the decreases in \% smoking and eGFR with aging were common characteristics in male and female patients. In addition, there were gender and aging differences in \% smoking, \% HT, BMI and eGFR, whereas no differences were observed in \% DM, \% dyslipidemia or \% HU.

Conclusions: Before $C A B G$, high-risk patients with coronary artery disease who is going to undergo $C A B G$ may need to be managed more strictly considering to gender and age to avoid CABG. (Cardiol J 2012; 19, 6: 618-624)

Key words: coronary artery disease, risk factor, coronary artery bypass grafting, gender, aging

Address for correspondence: Shin-ichiro Miura, MD, Fukuoka University School of Medicine, 7-45-1 Nanakuma, Jonan-ku, Fukuoka, 814-0180, Japan, tel: +81-92-801-1011, fax: +81-91-865-2692, e-mail: miuras@cis.fukuoka-u.ac.jp 


\section{Introduction}

The incidence of coronary artery disease $(\mathrm{CAD})$ is closely related to lifestyle, such as smoking, diet, and exercise [1, 2]. We have experienced a growing need for coronary artery bypass grafting (CABG) and percutaneous transluminal coronary angioplasty (PTCA) for CAD in younger patients because of the adoption of a western diet. Although CAD is a multifactorial disease that involves multiple loci and environmental factors [3, 4], it is possible to prevent $\mathrm{CAD}$ by aggressively eliminating the risk factors of CAD [5]. However, while the overall risk factors of $\mathrm{CAD}$ have been well studied, the risks of undergoing $\mathrm{CABG}$ are not known. The relative risks of $\mathrm{CAD}$ for each age group in females are generally lower than those in males, and the risk of death due to $\mathrm{CAD}$ in females is almost equal to that in males who are 10 years younger [3]. Thus, there may also be differences in the characteristics of patients who undergo $\mathrm{CABG}$ with regard to gender and age.

At the Department of Cardiovascular Surgery, Fukuoka University Hospital, CABG has been performed in about 1,500 cases over the past 15 years, and the patient characteristics have been recorded. Therefore, we evaluated the patient background in each age group for patients who underwent $\mathrm{CABG}$ and examined differences in gender and age in assessing the risk of CABG.

\section{Methods}

\section{Subjects}

We registered 1,525 patients who underwent CABG from April 1994 to December 2010 at the Department of Cardiovascular Surgery, Fukuoka University Hospital. Patients with Kawasaki disease $(\mathrm{n}=15)$ or familial hypercholesterolemia $(n=12)$ were excluded. The remaining 1,498 were used for further analysis.

The study was approved by the local ethic committee.

\section{Assessment of cardiovascular risk factors}

To analyze the influence of aging, the patients were divided into 5 groups according to age; $<50$ years (Group 1 - G1), 50-59 (G2), 60-69 (G3), 70-79 (G4) and $\geq 80$ (G5). In addition, to analyze the influence of gender, we further divided each of these groups into 2 groups (male and female). Cardiovascular risk factors (smoking, hypertension [HT], diabetes mellitus [DM], dyslipidemia [DL], hyperuricemia $[\mathrm{HU}]$, estimated glomerular filtration rate
[eGFR], low-density lipoprotein cholesterol [LDL-C], and body mass index [BMI]) were analyzed. We also analyzed the left ventricular ejection fraction (LVEF), left ventricular end-diastolic pressure (LVEDP) and EuroScore.

\section{Statistical analysis}

Statistical analysis was performed using the Stat View statistical software package (Stat View 5; SAS Institute INC., Cary, NC) at Fukuoka University. Data are expressed as the mean \pm standard deviation (SD). Categorical and continuous variables were compared by a $\chi^{2}$ analysis and analysis of variance followed by Fisher's PLSD, respectively. Multivariate analysis was performed using a logistic regression analysis for independent variables that were related to the gender. A value of $p<0.05$ was considered significant.

\section{Results}

Table 1 shows the patient characteristics for all of the patients and in male and female. Male showed significantly younger, higher percentages (\%) of smoking and HU, higher levels of serum creatinine $(\mathrm{Cr})$, and lower \% HT and DM, and lower levels of LVEF and EuroScore than female. Age is significantly associated with EuroScore because age over 60 years plus a score of 1 for every 5 years. These factors were associated with each other. Therefore, we excluded EuroScore from the logistic regression analysis and performed the analysis using independent variables (age, \% smoking, \% HT, $\% \mathrm{DM}, \% \mathrm{HU}$, serum $\mathrm{Cr}$, and LVEF) that were related to the gender (Table 2). All parameters identified independent variables associated with the gender difference.

Table 3 shows the patient characteristics for all of the patients and in G1-G5. The average ages for all patients, males and females were $67 \pm 9$, $66 \pm 10$ and $70 \pm 8$ years, respectively. Seventy-six percent of all patients were male and underwent GABG. The \% male with CABG decreased with aging. Further, BMI values in all patients, males, and females decreased with aging. $\%$ smoking in all patients, males and females was $21 \%, 25 \%$ and $7 \%$, respectively. $\%$ smoking in both males and females decreased with aging. $\%$ HT in all patients, males and females was $67 \%, 65 \%$ and $73 \%$, respectively. $\% \mathrm{HT}$ in all patients increased with aging. \% DL in all patients, males and females was $57 \%, 56 \%$ and $59 \%$, respectively. $\%$ DL in all patients and males decreased with aging. $\% \mathrm{DM}$ in all patients, males and females was $48 \%, 47 \%$, and $53 \%$, respectively. 
Table 1. Patient characteristics and physiological and biochemical parameters.

\begin{tabular}{lcccc}
\hline & All & Male & Female & $\begin{array}{c}\text { P (male } \\
\text { vs. female) }\end{array}$ \\
\hline $\mathrm{N}(\%)$ & 1498 & $1133(76 \%)$ & $365(24 \%)$ & $<0.001$ \\
Age (years) & $67 \pm 9$ & $66 \pm 10$ & $70 \pm 8$ & 0.480 \\
Body mass index $\left[\mathrm{kg} / \mathrm{m}^{2}\right]$ & $24 \pm 3$ & $24 \pm 3$ & $23 \pm 4$ & $<0.001$ \\
Smoking & $21 \%$ & $25 \%$ & $7 \%$ & 0.006 \\
Hypertension & $67 \%$ & $65 \%$ & $73 \%$ & 0.034 \\
Diabetes mellitus & $48 \%$ & $47 \%$ & $73 \%$ & $<0.001$ \\
Hyperuricemia & $11 \%$ & $14 \%$ & $4 \%$ & 0.386 \\
Dyslipidemia & $57 \%$ & $56 \%$ & $59 \%$ & 0.003 \\
Serum creatinine $[\mathrm{mg} / \mathrm{dL}]$ & $1.5 \pm 2.1$ & $1.6 \pm 2.3$ & $1.3 \pm 1.7$ & 0.696 \\
CKD (stage) & $2.7 \pm 0.9$ & $2.7 \pm 0.9$ & $2.6 \pm 1.0$ & 0.230 \\
eGFR [mL/min/1.73 mm $\left.{ }^{2}\right]$ & $57 \pm 23$ & $57 \pm 23$ & $56 \pm 24$ & 0.523 \\
LDL-cholesterol $[\mathrm{mg} / \mathrm{dL}]$ & $113 \pm 39$ & $112 \pm 38$ & $114 \pm 42$ & 0.670 \\
LVEP [mm Hg] & $14 \pm 7$ & $14 \pm 7$ & $13 \pm 7$ & $<0.001$ \\
Left ventricular ejection fraction (\%) & $59 \pm 16$ & $58 \pm 16$ & $62 \pm 15$ & 0.441 \\
Number of significant & $2.6 \pm 0.6$ & $2.6 \pm 0.6$ & $2.6 \pm 0.6$ & $<0.001$ \\
stenosed coronary vessels & & & & \\
EuroScore & $5.5 \pm 3.6$ & $5.1 \pm 3.6$ & $6.6 \pm 3.5$ & \\
\hline
\end{tabular}

CKD — chronic kidney disease; eGFR — estimated glomerular filtration rate; LDL - low-density lipoprotein; LVEDP — left ventricular end-diastolic pressure

Table 2. Independent variables that were related to the gender difference.

\begin{tabular}{lcc}
\hline Factors & $\begin{array}{c}\text { Odds ratio } \\
(\mathbf{9 5 \%} \text { Cl) }\end{array}$ & P \\
\hline Age (years) & $0.96(0.94-0.97)$ & $<0.001$ \\
Smoking (\%) & $3.96(2.53-6.19)$ & $<0.001$ \\
Hypertension (\%) & $0.72(0.54-0.95)$ & 0.020 \\
Diabetes mellitus (\%) & $0.67(0.52-0.87)$ & 0.003 \\
Hyperuricemia (\%) & $3.41(1.95-5.96)$ & $<0.001$ \\
Serum creatinine & $1.10(1.02-1.19)$ & 0.013 \\
[mg/dL] & $0.98(0.97-0.99)$ & $<0.001$ \\
Left ventricular & & \\
ejection fraction (\%) & & \\
\hline
\end{tabular}

There were significant changes in DM with regard to age in all patients and females.

Table 4 shows the physiological and biochemical parameters from G1 to G5 groups. eGFR in all patients, males and females was $57 \pm 23,57 \pm 23$ and $56 \pm 24 \mathrm{~mL} / \mathrm{min} / 1.73 \mathrm{~m}^{2}$, respectively, and there was no difference in eGFR between males and females (Table 4). The eGFR in all patients, males and females decreased with aging. The level of LDL-C in all patients, males and females was $113 \pm$ $\pm 39,112 \pm 38$, and $114 \pm 42 \mathrm{mg} / \mathrm{dL}$, respectively, and there was no difference in LDL-C with aging in all patients and females. However, the level of LDL-C in males were significantly decreased with aging. The LVEF in all patients, males and females was $59 \pm 16 \%, 58 \pm 16 \%$, and $62 \pm 15 \%$, respectively, and LVEF in females was significantly higher than that in males, except for G1. The EuroScore in all patients, males and females was $5.5 \pm 3.6,5.1 \pm$ \pm 3.6 , and $6.6 \pm 3.5$, respectively. The EuroScore in all patients, males and females increased with aging. There were no differences in LVEDP or the number of significantly stenosed coronary vessels with aging.

\section{Discussion}

In this contemporary data set, decreases in $\%$ smoking and eGFR with aging were common patient characteristics in both male and female patients. In addition, age, \% smoking, \% HT, \% DM, $\% \mathrm{HU}$, serum $\mathrm{Cr}$ and LVEF were related to the gender identified independent variables. Thus, high-risk patients with $\mathrm{CAD}$ who undergo $\mathrm{CABG}$ should be managed more strictly with regard to gender and age.

It has been reported that there are differences in the risk of CAD according to gender and age $[1,3]$. Patients background, such as age, $\%$ male, BMI, $\%$ HT, eGFR and EuroScore in this study were comparable to the Japanese patients group described in 
Table 3. Patient characteristics from G1 to G5 groups.

\begin{tabular}{|c|c|c|c|c|c|c|c|}
\hline & Group 1 & Group 2 & Group 3 & Group 4 & Group 5 & Total & $\begin{array}{l}P \text { for } \\
\text { trend }\end{array}$ \\
\hline \multicolumn{8}{|l|}{ All patients } \\
\hline $\mathrm{N}$ & 71 & 237 & 550 & 559 & 81 & 1498 & \\
\hline Age (years) & $44 \pm 5$ & $55 \pm 3$ & $65 \pm 3$ & $74 \pm 3$ & $82 \pm 2$ & $67 \pm 9$ & $<0.001$ \\
\hline Body mass index $\left[\mathrm{kg} / \mathrm{m}^{2}\right]$ & $25 \pm 4$ & $24 \pm 3$ & $24 \pm 3$ & $23 \pm 3$ & $22 \pm 3$ & $24 \pm 3$ & $<0.001$ \\
\hline Male & $94 \%$ & $85 \%$ & $79 \%$ & $69 \%$ & $57 \%$ & $76 \%$ & $<0.001$ \\
\hline Smoking & $39 \%$ & $33 \%$ & $23 \%$ & $13 \%$ & $7 \%$ & $21 \%$ & $<0.001$ \\
\hline Hypertension & $51 \%$ & $65 \%$ & $67 \%$ & $71 \%$ & $72 \%$ & $67 \%$ & 0.011 \\
\hline Diabetes mellitus & $47 \%$ & $53 \%$ & $51 \%$ & $46 \%$ & $36 \%$ & $48 \%$ & 0.036 \\
\hline Hyperuricemia & $17 \%$ & $15 \%$ & $11 \%$ & $10 \%$ & $7 \%$ & $11 \%$ & 0.064 \\
\hline Dyslipidemia & $70 \%$ & $67 \%$ & $53 \%$ & $55 \%$ & $52 \%$ & $57 \%$ & 0.004 \\
\hline \multicolumn{8}{|l|}{ Male } \\
\hline $\mathrm{N}$ & 67 & 202 & 433 & 385 & 46 & 1133 & \\
\hline Age [years] & $44 \pm 5$ & $55 \pm 3$ & $65 \pm 3$ & $74 \pm 3$ & $82 \pm 2$ & $66 \pm 10$ & $<0.001$ \\
\hline Body mass index $\left[\mathrm{kg} / \mathrm{m}^{2}\right]$ & $25 \pm 4$ & $24 \pm 3$ & $24 \pm 3$ & $23 \pm 3$ & $23 \pm 2$ & $24 \pm 3$ & $<0.001$ \\
\hline Smoking & $39 \%$ & $35 \%$ & $27 \%$ & $18 \%$ & $11 \%$ & $25 \%$ & $<0.001$ \\
\hline Hypertension & $51 \%$ & $64 \%$ & $66 \%$ & $68 \%$ & $63 \%$ & $65 \%$ & 0.091 \\
\hline Diabetes mellitus & $43 \%$ & $51 \%$ & $49 \%$ & $45 \%$ & $35 \%$ & $47 \%$ & 0.229 \\
\hline Hyperuricemia & $16 \%$ & $17 \%$ & $13 \%$ & $12 \%$ & $11 \%$ & $14 \%$ & 0.393 \\
\hline Dyslipidemia & $70 \%$ & $67 \%$ & $52 \%$ & $53 \%$ & $48 \%$ & $56 \%$ & $<0.001$ \\
\hline \multicolumn{8}{|l|}{ Female } \\
\hline $\mathrm{N}$ & 4 & 35 & 117 & 174 & 35 & 365 & \\
\hline Age [years] & $44 \pm 4$ & $55 \pm 3$ & $65 \pm 3$ & $74 \pm 3$ & $83 \pm 2$ & $70 \pm 8$ & $<0.001$ \\
\hline Body mass index $\left[\mathrm{kg} / \mathrm{m}^{2}\right]$ & $21 \pm 4$ & $24 \pm 4$ & $24 \pm 4$ & $23 \pm 3$ & $22 \pm 3$ & $23 \pm 4$ & $<0.001$ \\
\hline Smoking & $50 \%$ & $20 \%$ & $6 \%$ & $4 \%$ & $3 \%$ & $7 \%$ & $<0.001$ \\
\hline Hypertension & $50 \%$ & $66 \%$ & $70 \%$ & $75 \%$ & $83 \%$ & $73 \%$ & 0.317 \\
\hline Diabetes mellitus & $100 \%$ & $66 \%$ & $60 \%$ & $48 \%$ & $37 \%$ & $53 \%$ & 0.011 \\
\hline Hyperuricemia & $25 \%$ & $3 \%$ & $4 \%$ & $4 \%$ & $3 \%$ & $4 \%$ & 0.317 \\
\hline Dyslipidemia & $75 \%$ & $66 \%$ & $56 \%$ & $59 \%$ & $57 \%$ & $59 \%$ & 0.795 \\
\hline
\end{tabular}

previous reports [6-8]. Our patients would not be in a specific population. Based on the present data, among patients with $\mathrm{CABG}$, the difference in age between males and females was only 4 years (the average age in males and females was $66 \pm 10$ and $70 \pm 8$ years, respectively), while the age of onset of CAD in females is almost 10 years younger than that in males [3]. The criteria for patients to undergo CABG according to Japanese guidelines were a repeated coronary restenosis after PTCA in addition to three-vessel coronary artery disease and significant coronary stenosis in the left main trunk [9]. CABG is generally considered the treatment of choice for patients with severe stenotic lesions. Females with CAD are less likely than males to have typical chest pain as a chief complaint. Herlitz et al. [10] and Milner et al. [11] reported that, among females, symptoms of myocardial infarction often include back pain, abdominal pain and vomiting in addition to, or instead of, chest pain. The diagnosis of CAD in females is usually delayed compared to that in males. Female patients may have been considered to be candidates for CABG because they were in a more severe state. Thus, males may be more likely to receive PTCA while females are selected for CABG.

Although we did not analyze the influence of menopause, the suppression of atherosclerosis by estrogen reduces the incidence of $\mathrm{CAD}$ in younger females. The risk of CAD in menopausal females may be comparable to that in males. The ability of estrogen to improve glucose and lipid metabolism and its antihypertensive action [12], in addition to its direct effects on the vessel wall [13] for preventing $\mathrm{CAD}$, may be lost due to aging and menopause. Thus, further studies are needed to confirm that females who underwent CABG were older than males because of menopause. 
Table 4. Physiological and biochemical parameters from G1 to G5 groups.

\begin{tabular}{|c|c|c|c|c|c|c|c|}
\hline & Group 1 & Group 2 & Group 3 & Group 4 & Group 5 & Total & $\begin{array}{l}P \text { for } \\
\text { trend }\end{array}$ \\
\hline \multicolumn{8}{|l|}{ All patients } \\
\hline $\mathrm{N}$ & 71 & 237 & 550 & 559 & 81 & 1498 & \\
\hline Serum creatinine $[\mathrm{mg} / \mathrm{dL}]$ & $1.8 \pm 2.9$ & $2.0 \pm 3.1$ & $1.4 \pm 1.9$ & $1.5 \pm 1.9$ & $1.2 \pm 0.8$ & $1.5 \pm 2.1$ & 0.003 \\
\hline CKD (stage) & $2.3 \pm 1.0$ & $2.5 \pm 1.1$ & $2.6 \pm 0.9$ & $2.8 \pm 0.8$ & $2.8 \pm 0.7$ & $2.7 \pm 0.9$ & $<0.001$ \\
\hline eGFR $\left[\mathrm{mL} / \mathrm{min} / 1.73 \mathrm{~mm}^{2}\right]$ & $68 \pm 26$ & $62 \pm 28$ & $60 \pm 23$ & $52 \pm 19$ & $51 \pm 19$ & $57 \pm 23$ & $<0.001$ \\
\hline LDL-cholesterol [mg/dL] & $113 \pm 45$ & $112 \pm 37$ & $116 \pm 39$ & $110 \pm 39$ & $111 \pm 31$ & $113 \pm 39$ & 0.092 \\
\hline LVEDP [mm Hg] & $15 \pm 7$ & $15 \pm 8$ & $13 \pm 7$ & $14 \pm 7$ & $13 \pm 7$ & $14 \pm 7$ & 0.259 \\
\hline $\begin{array}{l}\text { Left ventricular ejection } \\
\text { fraction }(\%)\end{array}$ & $55 \pm 17$ & $57 \pm 17$ & $59 \pm 15$ & $60 \pm 15$ & $60 \pm 17$ & $59 \pm 16$ & 0.040 \\
\hline $\begin{array}{l}\text { Number of significant } \\
\text { stenosed coronary vessels }\end{array}$ & $2.5 \pm 0.7$ & $2.6 \pm 0.6$ & $2.6 \pm 0.6$ & $2.6 \pm 0.6$ & $2.6 \pm 0.6$ & $2.6 \pm 0.6$ & 0.052 \\
\hline EuroScore & $2.8 \pm 2.9$ & $3.0 \pm 3.1$ & $4.7 \pm 3.1$ & $7.1 \pm 3.2$ & $8.7 \pm 3.5$ & $5.5 \pm 3.6$ & $<0.001$ \\
\hline \multicolumn{8}{|l|}{ Male } \\
\hline $\mathrm{N}$ & 67 & 202 & 433 & 385 & 46 & 1133 & \\
\hline Serum creatinine $[\mathrm{mg} / \mathrm{dL}]$ & $1.8 \pm 2.9$ & $2.1 \pm 3.2$ & $1.4 \pm 1.9$ & $1.6 \pm 2.0$ & $1.3 \pm 0.9$ & $1.6 \pm 2.3$ & 0.005 \\
\hline CKD (stage) & $2.4 \pm 1.0$ & $2.6 \pm 1.1$ & $2.6 \pm 0.9$ & $2.8 \pm 0.8$ & $2.8 \pm 0.7$ & $2.7 \pm 0.9$ & $<0.001$ \\
\hline eGFR $\left[\mathrm{mL} / \mathrm{min} / 1.73 \mathrm{~mm}^{2}\right]$ & $67 \pm 26$ & $60 \pm 27$ & $60 \pm 22$ & $52 \pm 20$ & $51 \pm 18$ & $57 \pm 23$ & $<0.001$ \\
\hline LDL-cholesterol [mg/dL] & $115 \pm 46$ & $112 \pm 37$ & $117 \pm 39$ & $108 \pm 37$ & $108 \pm 27$ & $112 \pm 38$ & 0.017 \\
\hline LVEDP [mm Hg] & $15 \pm 7$ & $15 \pm 8$ & $13 \pm 7$ & $14 \pm 7$ & $12 \pm 6$ & $14 \pm 7$ & 0.078 \\
\hline $\begin{array}{l}\text { Left ventricular ejection } \\
\text { fraction }(\%)\end{array}$ & $55 \pm 17$ & $55 \pm 17$ & $59 \pm 15$ & $59 \pm 15$ & $59 \pm 15$ & $58 \pm 16$ & 0.053 \\
\hline $\begin{array}{l}\text { Number of significant } \\
\text { stenosed coronary vessels }\end{array}$ & $2.5 \pm 0.7$ & $2.6 \pm 0.7$ & $2.6 \pm 0.6$ & $2.6 \pm 0.6$ & $2.7 \pm 0.6$ & $2.6 \pm 0.6$ & 0.183 \\
\hline EuroScore & $2.9 \pm 3.0$ & $3.0 \pm 3.2$ & $4.5 \pm 3.1$ & $6.8 \pm 3.2$ & $8.4 \pm 3.7$ & $5.1 \pm 3.6$ & $<0.001$ \\
\hline \multicolumn{8}{|l|}{ Female } \\
\hline N & 4 & 35 & 117 & 174 & 35 & 365 & \\
\hline Serum creatine $[\mathrm{mg} / \mathrm{dL}]$ & $0.7 \pm 0.1$ & $1.2 \pm 2.0$ & $1.4 \pm 1.9$ & $1.2 \pm 1.6$ & $1.0 \pm 0.7$ & $1.3 \pm 1.7$ & 0.808 \\
\hline CKD (stage) & $1.8 \pm 0.5$ & $2.2 \pm 1.0$ & $2.5 \pm 1.1$ & $2.8 \pm 0.8$ & $2.8 \pm 0.8$ & $2.6 \pm 1.0$ & 0.006 \\
\hline eGFR $\left[\mathrm{mL} / \mathrm{min} / 1.73 \mathrm{~mm}^{2}\right]$ & $81 \pm 20$ & $71 \pm 31$ & $59 \pm 26$ & $51 \pm 19$ & $50 \pm 20$ & $56 \pm 24$ & $<0.001$ \\
\hline LDL-cholesterol [mg/dl] & $82 \pm 20$ & $112 \pm 35$ & $115 \pm 48$ & $114 \pm 40$ & $116 \pm 36$ & $114 \pm 42$ & 0.633 \\
\hline LVEDP [mm Hg] & $13 \pm 6$ & $12 \pm 6$ & $13 \pm 7$ & $14 \pm 7$ & $15 \pm 7$ & $13 \pm 7$ & 0.728 \\
\hline $\begin{array}{l}\text { Left ventricular ejection } \\
\text { fraction }(\%)\end{array}$ & $51 \pm 13$ & $67 \pm 12$ & $62 \pm 14$ & $62 \pm 14$ & $61 \pm 19$ & $62 \pm 15$ & 0.145 \\
\hline $\begin{array}{l}\text { Number of significant } \\
\text { stenosed coronary vessels }\end{array}$ & $2.3 \pm 1.0$ & $2.6 \pm 0.6$ & $2.6 \pm 0.6$ & $2.5 \pm 0.7$ & $2.6 \pm 0.6$ & $2.6 \pm 0.6$ & 0.561 \\
\hline EuroScore & $1.8 \pm 0.5$ & $3.1 \pm 2.8$ & $5.3 \pm 2.8$ & $7.7 \pm 3.2$ & $9.2 \pm 3.2$ & $6.6 \pm 3.5$ & $<0.001$ \\
\hline
\end{tabular}

Abbreviations as in Table 1.

Next, we address the effects of aging between males and females for each coronary risk factor for undergoing CABG. \% smoking and \% DL significantly decreased and \% HT increased with aging. The incidence of smoking among Japanese peaks in the $30 \mathrm{~s}$ among both males and females, and tends to decline with aging. Smoking contributes to the development of atherosclerosis and directly impairs vascular endothelial cells. In addition, smoking increases heart rate and blood pressure by stimulating sympathetic nerves [14], in addition to coagu- lation factors and platelet function [15]. Epidemiological studies have clearly shown that smoking significantly increases the morbidity and mortality of CAD [16]. Although older patients who underwent CABG were expected to have higher rates of smoking than younger patients, $\%$ smoking significantly decreased with aging. The reason for this result is unknown. Once patient has CAD, they may stop smoking and then undergo CABG. It is important to prevent smoking before it starts, especially in adolescence. However, this registry did not analyze 
the number of cigarettes smoked or the duration of smoking.

$\% \mathrm{DL}$ is high among males before age 50 , and becomes high in females after age 50 [17]. However, in this registry, the rate was similar for both males and females in each generation. Since \% DL in females in Group G1 + G2 (age $<60$ years) was relatively high, premenopausal females with $\mathrm{DL}$ disproportionately underwent CABG. Premenopausal females with lipid disorders are likely to require aggressive medical therapy. In the present study, other lipid profiles, such as triglyceride, high-density lipoprotein cholesterol (HDL-C), non-HDL-C, and the ratio of LDL-C to HDL-C, were not evaluated, and further studies are needed.

While DM is a risk factor for CAD, we could not conclude that $\mathrm{DM}$ is a risk factor in gender or age for CABG. Coronary stenotic lesions in patients with DM generally show a small vessel diameter and longer length, and the long-term prognosis of percutaneous coronary intervention is worse than that of CABG. The onset of DM at a younger age is associated with severe $\mathrm{CAD}$, and will require CABG. Although there were no differences in $\% \mathrm{DM}$ with regard to gender or age, the risk for $\mathrm{CAD}$ death in females with DM is 1.5 times higher than that in males $[18,19]$.

The prevalence of HT is known to vary with age in both males and females. In males, it increases with age from the $30 \mathrm{~s}$, peaks at around 70, and remains constant thereafter. Meanwhile, the prevalence of $\mathrm{HT}$ in females increases rapidly after age 40 , and the prevalence in females at age 70 is similar to that in males [20]. \% HT in all patients increased with aging in this study. This means that HT may not be a particular risk factor for CABG. However, eGFR was associated with HT, and the values decreased with aging, which indicated that older patients may require renal protection.

The EuroScore is useful for determining the risks associated with CABG [21] and is routinely used in clinical practice in Japan. Age (over 60 years plus a score of 1 for every 5 years) and gender (female plus a score of 1) are also included in the evaluation. In this study, the EuroScore increased with aging in both males and females, and the score in females was significantly higher than that in males. Currently, the Japan SCORE is being evaluated for the preoperative assessment of risk for cardiac surgery using the Japan Cardiovascular Surgery Database.

\section{Limitations of the study}

This study has several limitations. First, this is a single-center registry in patients who under- went $\mathrm{CABG}$. Second, CABG is performed far less often in Japan than in Western countries, for several reasons: 1) Japanese hate surgery, 2) the difference in the number of physicians and surgeons, and 3 ) the severity of atherosclerosis. We did not take into account these factors in this registry. Third, although the relation between aging and the prevalence of hypertension seems to be more obvious in females than in males, \% HT in females did not significantly increase with aging. In addition, lower LDL-C levels were observed in female-G1. These observations may be due to small sample size of female-G1 and -G5. Forth, this registry is a retrospective evaluation of the past 15 years, and the therapeutic strategies used in recent patients may be different from those in patients at the initial registration. Finally, we did not analyze the differences in patient characteristics between $\mathrm{CAD}$ patients with and without CABG.

\section{Conclusions}

Decreases in \% smoking and eGFR with aging were common patient characteristics in both male and female patients. Age, \% smoking, \% HT, \% DM, $\% \mathrm{HU}$, serum $\mathrm{Cr}$ and LVEF were related to the gender identified independent variables. Therefore, before CABG, high-risk patients with $\mathrm{CAD}$ who is going to undergo $\mathrm{CABG}$ may need to be managed more strictly considering to gender and age to avoid CABG.

Conflict of interest: none declared

\section{References}

1. Teramoto T, Sasaki J, Ueshima H et al. Risk factors of atherosclerotic diseases. Executive summary of Japan Atherosclerosis Society (JAS) guideline for diagnosis and prevention of atherosclerosis cardiovascular diseases for Japanese. J Atheroscler Thromb, 2007; 14: 267-277.

2. Yagi H, Komukai K, Hashimoto $\mathrm{K}$ et al. Difference in risk factors between acute coronary syndrome and stable angina pectoris in the Japanese: smoking as a crucial risk factor of acute coronary syndrome. J Cardiol, 2010; 55: 345-353.

3. Lawton JS. Sex and gender differences in coronary artery disease. Semin Thorac Cardiovasc Surg, 2011; 23: 126-130.

4. Atkov OY, Gorokhova SG, Sboev AG et al. Coronary heart disease diagnosis by artificial neural networks including genetic polymorphisms and clinical parameters. J Cardiol, 2012; 59: 190-194.

5. Nakamura M, Yamashita T, Yajima J et al.; Shinken Database Study Group. Impact of early statin initiation on secondary prevention in Japanese patients with coronary artery disease. J Cardiol, 2011; 57: 172-180.

6. Kinoshita T, Asai T, Murakami Y, Suzuki T, Kambara A, Matsubayashi K. Preoperative renal dysfunction and mortality after off-pump coronary artery bypass grafting in Japanese. Circ J, 2010; 74: 1866-1872. 
7. Orii K, Hioki M, Iedokoro Y, Shimizu K. Prognostic factors affecting clinical outcomes after coronary artery bypass surgery: Analysis of patients with chronic kidney disease after 5.9 years of follow-up. J Nihon Med Sch, 2011; 78: 156-165.

8. Fukui T, Takanashi S. Gender differences in clinical and angiographic outcomes after coronary artery bypass surgery. Circ J, 2010; 74: 2103-2108.

9. Kiyamura S. Guidelines for the clinical application of bypass grafts and the surgical techniques. Circ J, 2006; 70 (suppl. VI): 1477-1553.

10. Herlitz J, Bång A, Karlson BW, Hartford M. Is there a gender difference in aetiology of chest pain and symptoms associated with acute myocardial infarction? Eur J Emerg Med, 1999; 6: 311-315.

11. Milner KA, Funk M, Richards S et al. Gender differences in symptom presentation associated with coronary heart disease. Am J Cardiol, 1999; 84: 396-339.

12. Lobo RA. Benefits and risks of estrogen replacement therapy. Am J Obstet Gynecol, 1995; 173: 982-989.

13. Krasinski K, Spyridopoulos I, Asahara T, van der Zee R, Isner JM, Losordo DW. Estradiol accelerates functional endothelial recovery after arterial injury. Circulation, 1997; 95: 1768-1772.

14. Cryer PE, Haymond MW, Santiago JV, Shah SD. Norepinephrine and epinephrine release and adrenergic mediation of smoking-associated hemodynamic and metabolic events. N Engl J Med, 1976; 295: 573-577.
15. Zidovetzki R, Chen P, Fisher M, Hofman FM, Faraci FM. Nicotine increases plasminogen activator inhibitor-1 production by human brain endothelial cells via protein kinase cûassociated pathway. Stroke, 1999; 30: 651-655.

16. Benowitz NL. Cigarette smoking and cardiovascular disease: Pathophysiology and implications for treatment. Prog Cardiovasc Dis, 2003; 46: 91-111.

17. Thom T, Haase N, Rosamond W et al.; American Heart Association Statistics Committee and Stroke Statistics Subcommittee. Heart disease and stroke statistics — 2006 update: A report from the American Heart Association Statistics Committee and Stroke Statistics Subcommittee. Circulation, 2006; 113: e85-e151.

18. Lee WL, Cheung AM, Cape D, Zinman B. Impact of diabetes on coronary artery disease in women and men: a meta-analysis of prospective studies. Diabetes Care, 2000; 23: 962-968.

19. Huxley R, Barzi F, Woodward M. Excess risk of fatal coronary heart disease associated with diabetes in men and women: metaanalysis of 37 prospective cohort studies. BMJ, 2006; 332: 73-78.

20. Staessen JA, Bieniaszewski L, O’Brien ET, Imai Y, Fagard R. An epidemiological approach to ambulatory blood pressure monitoring: The Belgian Population Study. Blood Press Monit, 1996; 1: $13-26$.

21. Nashef SAM, Roques F, Michel P, Gauducheau E, Lemeshow S, Salamon R, The EuroSCORE study Group. European system for cardiac risk evaluation (EuroSCORE). Eur J Cardiothorac Surg, 1999; 16: 9-13. 